\title{
Naphthalene substituted benzo[c]coumarins: Synthesis, characterization and evaluation of antibacterial activity and cytotoxicity
}

https://doi.org/10.1515/hc-2019-0024

Received July 16, 2019; accepted November 07, 2019.

Abstract: Novel congeners of naphthalene substituted benzo[c]coumarins (2a-f) were synthesized by reaction of various 3-coumarinoyl methyl pyridinium bromide salts (1a-d) with a selected set of acetyl naphthalene in the presence of sodium acetate in refluxing glacial acetic acid. Structures of the synthesized compounds were confirmed by elemental analysis and by various spectroscopic techniques such as ${ }^{1} \mathrm{H}-\mathrm{NMR},{ }^{13} \mathrm{C}$-NMR, DEPT, and MS spectral data. Synthesised compounds were screened for antibacterial activity and cytotoxicity against different human cancer cell lines including cervix cancer (HeLa), breast cancer (MCF-7) and lung cancer (A549) using tetrazolium dye 3-(4,5-dimethylthiazol-2-yl)-2,5-diphenyltetrazolium bromide (MTT) cell viability assay. Although with varying degrees, a significant growth inhibitory and cytotoxic effects were observed on all three cancer cell lines. Compounds $\mathbf{2 b}$ and $\mathbf{2 e}$ showed significant growth inhibitory and cytotoxicity against aforementioned cancer cell lines.

Keywords: Benzo[c]coumarins, Naphthalene, Antibacterial, Cytotoxicity.

\section{Introduction}

Coumarin is known as benzopyran-2-one. This flavonoid class of compound is naturally occurring and has a diverse array of biochemical and pharmacological activities with low toxicity [1]. Coumarins and their analogs

\footnotetext{
* Corresponding author: Kaushal Patel, Department of Advanced Organic Chemistry, P. D. Patel Institute of Applied Sciences, Charotar University of Science and Technology, Gujarat 388421, India, email: kaus_chem@yahoo.com Mrugesh Patel, Department of Advanced Organic Chemistry, P. D. Patel Institute of Applied Sciences, Charotar University of Science and Technology, Gujarat 388421, India
}

are found in a wide range of pharmaceutically active compounds such as novobiocin, clorobiocin etc. These analogs serve as potent inhibitors of bacterial DNA gyrase [2]. Whereas, osthole [3] and esculetin [4] have been reported to inhibit the growth of cancer cells. Coumarins also possess significant biological activities such as antimicrobial [5], anticancer [6], antioxidant [7], antiviral [8] and anti-tuberculosis [9]. Coumarin compounds exert diverse mechanisms of action on many biological targets for cancer oncology [10]. Benzo[c]coumarin derivatives such as autumnariol [11], autumnariniol, alternariol [12], ellagic acid and altenuisol [13] can be found in several natural products as well as in synthetic ones. They exhibit a wide range of pharmacological activities such as antimicrobial [14] and anti-tumor [15] etc. Furthermore, several naphthalene derivatives are found to be potent anticancer agents [16], antimicrobial agents [17], antioxidant [18] and anti-inflammatory [19] agents.

Thus, we anticipated that the combination of benzo[c]coumarin with substituted naphthalene nucleus would allow the development of a new class of biologically active molecules. In continuation of our interest in synthesizing novel modified fused chromenone derivatives [20], it was thought worthwhile to incorporate the naphthalene nucleus into the coumarin moiety as a substituent group. Here, we report the synthesis of various naphthalene substituted benzo[c]coumarins (2a-f) (Scheme 1). The synthesized compounds were investigated for their antibacterial activity and cytotoxicity on A549 human lung adenocarcinoma, HeLa human cervical adenocarcinoma, and MCF-7 human breast adenocarcinoma cell lines. This strategy was found useful to simultaneously address multiple biological targets in treatments of multiple diseases.

\section{Results and discussion}

A simple and efficient method for the synthesis of coumarin-naphthalene derivatives was developed and 
evaluated for cytotoxicity as well as anti-bacterial activity. Coumarin-naphthalene conjugates were synthesized using the route illustrated in Scheme 1. The condensation between 3-coumarinoyl pyridinium bromide salts and acetyl naphthalenes in the presence of catalytic amount of sodium acetate in acetic acid resulted in the formation of the targeted final compounds. All of the synthesized coumarin-naphthalene derivatives were characterized using NMR, IR and MS.

Anti-bacterial Activity: All the synthesized compounds were screened for their in vitro antibacterial activity against two Gram-positive organisms, Bacillus subtilis (MTCC 441) and Staphylococcus aureus (MTCC96), and two Gram-negative organisms, Escherichia coli (MTCC 443) and Proteus Vulgaris (MTCC 1771). For comparison, Streptomycin was used as a standard drug.

The antibacterial screening of the tested compounds (2a-2f) showed that they had significant activity against Gram-positive as well as Gram-negative bacteria. Compound $\mathbf{2 b}$ and $\mathbf{2 e}$ showed remarkable activity. While compound $\mathbf{2 c}$ and $\mathbf{2 f}$ showed moderate activity. In comparison, compound $\mathbf{2 a}$ and $\mathbf{2 d}$ exhibited partial activity. Compounds possessing a methoxy group on the $8^{\text {th }}$ position of coumarin were shown to have a significant effect on activity. The values of MIC against pathogenic microorganisms are depicted in Table 1.

Cytotoxicity Activity: In vitro growth inhibitory activity of synthesized compounds was performed on three cell lines HeLa (human cervix cancer cell line), MCF-7 (Human breast cancer cell line) and A549 (human lung cancer cell line). $\mathrm{IC}_{50}$ (concentration of compound

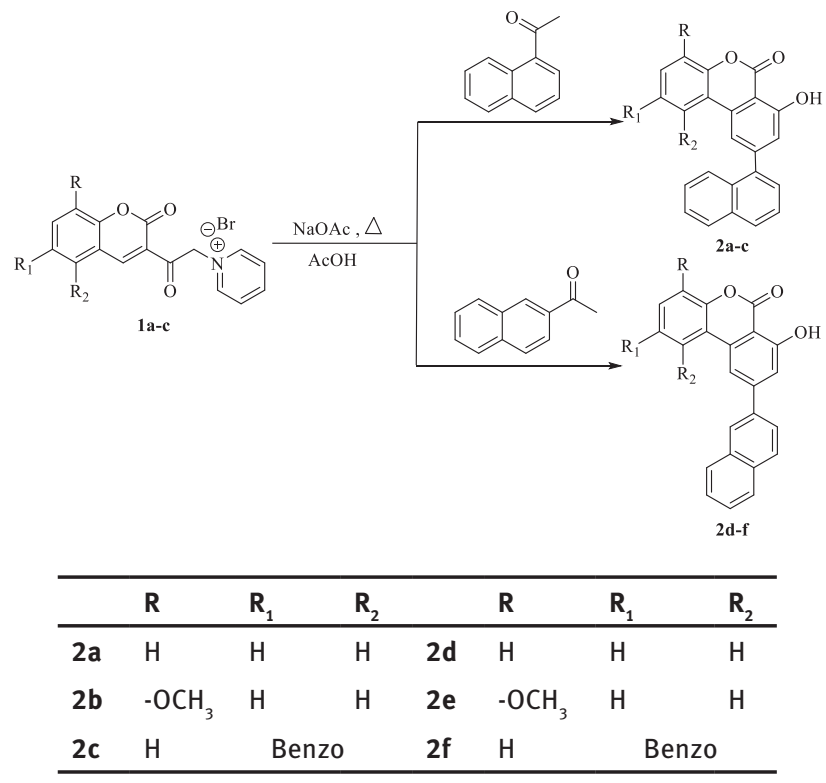

Scheme 1. causing 50\% cell population death) was calculated from the dose-reponce curve. The dose-responce curve was generated using values of different concentrations of compounds and their responce obtained from cell lines. The results (Table 2) obtained were compared with the reference drug cis-platin under similar experimental conditions using colorimetric MTT assay and this has been considered as a positive control. The dose-response curves of all the compounds for cell lines MCF-7, A549 and HeLa (Figure 1) were generated by plotting percentage Inhibition (cytotoxicity) against the concentration of compounds.

Cytotoxicity was expressed as the mean $\mathrm{IC}_{50}$ of three independent experiments. All the synthesized compounds exhibited good cytotoxicity, with $\mathrm{IC}_{50}$ values in the range 18-55 $\mu \mathrm{M}$. Compound $\mathbf{2 b}$ and $\mathbf{2 e}$, which possessed a methoxy group on the $8^{\text {th }}$ position of coumarin, exhibited excellent cytotoxicity against three cell lines MCF-7 $\left(\mathrm{IC}_{50}: 23.54 \pm 2.00,22.33 \pm 0.91 \mu \mathrm{M}\right), \mathrm{A} 549$ $(26.19 \pm 0.87,20.44 \pm 1.13 \mu \mathrm{M})$ and HeLa $(28.58 \pm 0.28$, $18.57 \pm 0.31 \mu \mathrm{M})$.

Whereas, compound 2c and 2f, which contained a fused benzene ring on the 5,6-position of coumarin, showed good activity against MCF-7 ( IC $_{50}: 28.87 \pm 1.63$, $26.22 \pm 0.34 \mu \mathrm{M}), \mathrm{A} 549(27.78 \pm 0.85,26.54 \pm 1.70 \mu \mathrm{M})$ and HeLa $(35.33 \pm 1.23,26.99 \pm 1.05 \mu \mathrm{M})$ and the remaining

Table 1 The antibacterial activities of synthesized compounds expressed as minimum inhibitory concentration $(\mu \mathrm{g} / \mathrm{mL})$

\begin{tabular}{lcccccc}
\hline \multirow{2}{*}{ Compound } & \multicolumn{2}{c}{ Gram-positive } & & \multicolumn{2}{c}{ Gram-negative } \\
\cline { 2 - 3 } \cline { 5 - 6 } & B. subtilis & S. aureus & & E. coli & P. vulgaris \\
\hline $\mathbf{2 a}$ & $>100$ & $>100$ & & $>100$ & $>100$ \\
$\mathbf{2 b}$ & 25 & $>100$ & & 25 & 50 \\
$\mathbf{2 c}$ & 50 & 50 & & $>100$ & $>100$ \\
$\mathbf{2 d}$ & $>100$ & $>100$ & & $>200$ & 50 \\
$\mathbf{2 e}$ & 25 & 50 & & 50 & 12.5 \\
$\mathbf{2 f}$ & 50 & $>100$ & & 50 & $>100$ \\
Streptomycin & 12.5 & 12.5 & & 12.5 & 6.25 \\
\hline
\end{tabular}

Table 2 In vitro cytotoxicity of compounds (2a-2f) evaluated on Human cancer cell lines MCF-7, A549 and HeLa [IC $\left.{ }_{50}(\mu M)\right]$.

\begin{tabular}{lccc}
\hline Compound & MCF-7 & A549 & HeLa \\
\hline 2a & $38.53 \pm 1.48$ & $37.29 \pm 1.54$ & $42.96 \pm 0.36$ \\
2b & $23.54 \pm 2.00$ & $26.19 \pm 0.87$ & $28.58 \pm 0.28$ \\
2c & $28.87 \pm 1.63$ & $27.78 \pm 0.85$ & $35.33 \pm 1.23$ \\
2d & $39.87 \pm 1.62$ & $47.17 \pm 1.15$ & $55.59 \pm 0.96$ \\
2e & $22.33 \pm 0.91$ & $20.44 \pm 1.13$ & $18.57 \pm 0.31$ \\
2f & $26.22 \pm 0.34$ & $26.54 \pm 1.70$ & $26.99 \pm 1.05$ \\
Cisplatin & $17.59 \pm 1.36$ & $11.72 \pm 0.70$ & $7.75 \pm 0.42$ \\
\hline
\end{tabular}

Mean \pm S.D.; values are means of three independent experiments. 

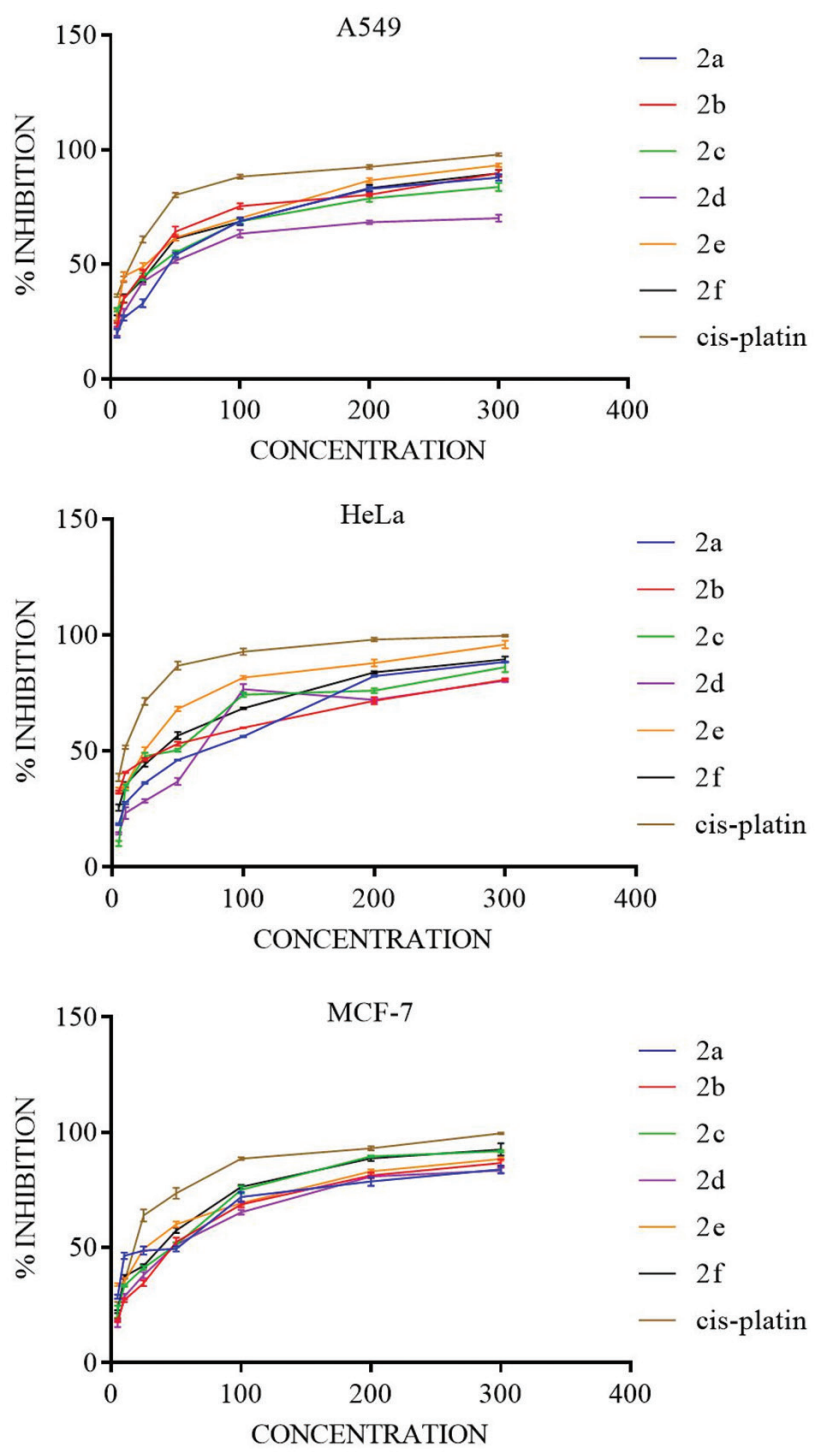

Figure 1. The dose-response curve showing the in vitro inhibitory activity of the compounds (2a-2f) against A549, MCF-7 and HeLa cell lines.

compounds exhibited moderate activity MCF-7 $\left(\mathrm{IC}_{50}: 38.53 \pm 1.48,39.87 \pm 1.62 \mu \mathrm{M}\right)$, A5 $49(37.29 \pm 1.54$, $47.17 \pm 1.15 \mu \mathrm{M})$ and HeLa $(42.96 \pm 0.36,55.59 \pm 0.96 \mu \mathrm{M})$.

\section{Conclusion}

The present study deals with a novel and efficient route for the synthesis of Naphthalene substituted benzo[c] coumarin analogues. The spectral characterization of the compounds confirmed their chemical structures. The results showed that some of the compounds possessed good antibacterial properties as well as good cytotoxicity against several cancer cell lines. Most of the compounds are promising for further investigation to develop a new chemical class of drugs which act as antibacterial and anticancer agents.

\section{Experimental Details}

\section{Materials and Methods}

All the reagents, solvents and starting materials were commercially procured from Loba Chemie, Merck, and Sigma Aldrich. They were used without further purification. Melting points were recorded (uncorrected) on capillary melting point apparatus. Thin-layer chromatography (TLC, on aluminum plates coated with silica gel $60 \mathrm{~F}_{254}, 0.25 \mathrm{~mm}$ thickness, Merck) was used for monitoring the progress of reactions, purity, and homogeneity of the synthesized compounds. Elemental analysis (\% C, H, N) was carried out on Perkin-Elmer 2400 series-II elemental analyzer. The FTIR spectra were recorded using potassium bromide disc on a Thermo Scientific Nicolet 6700 spectrophotometer and only the characteristic peaks are reported. ${ }^{1} \mathrm{H}-\mathrm{NMR}$ and ${ }^{13} \mathrm{C}-\mathrm{NMR}$ spectra were recorded using DMSO- $d_{6}$ and $\mathrm{CDCl}_{3}$ solvents on Bruker Avance 400 (MHz) spectrometer using solvent peak as an internal standard at 400 and $100 \mathrm{MHz}$, respectively. Chemical shifts are reported in parts per million ( $\mathrm{ppm})$. Mass spectra were documented on Shimadzu QP2010 Spectrometer. 3-coumarinoyl methyl pyridinium bromide salts (1a-1c) were prepared according to the literature procedures [20].

General procedure for the synthesis of Naphthalene substituted benzo[c]coumarins (2a-f): In a round bottom flask $(100 \mathrm{~mL})$, a solution of appropriate acetyl naphthalene (1-acetyl naphthalene/2-acetyl naphthalene) ( $0.0058 \mathrm{~mol}$ ) was taken in glacial acetic acid $(15 \mathrm{~mL})$. To this solution, sodium acetate $(0.06 \mathrm{~mol})$ and an appropriate 3-coumarinoyl methyl pyridinium bromide salt (1a-1c) $(0.006 \mathrm{~mol})$ in acetic acid $(10 \mathrm{~mL})$ were added with stirring. The reaction mixture was stirred at room temperature for 45 minutes and then refluxed in an oil bath at $140-145^{\circ} \mathrm{C}$ for 10 hours. The reaction progress was monitored using TLC. It was then poured in water $(75 \mathrm{~mL})$ and the crude solid obtained was extracted with chloroform (3 x $50 \mathrm{~mL}$ ). The organic layer was washed with $10 \%$ aqueous sodium bicarbonate solution $(50 \mathrm{~mL}$ ), water $(50 \mathrm{~mL})$ and dried over anhydrous sodium sulfate. Distillation of chloroform under vacuum gave a gummy 
material which was subjected to column chromatography using ethyl acetate : pet.ether $\left(60-80^{\circ} \mathrm{C}\right)$ in the ratio of $(2: 8)$ as an eluent to afford the product (2a-f) respectively.

\section{Characterization data of compounds $2 a-2 f$}

7-hydroxy-9-(naphthalen-1-yl)-6H-benzo[c]chromen6-one (2a): Yield $=67 \%$ white crystalline solid. m.p. = 190-192 ${ }^{\circ} \mathrm{C}$. Selected IR frequencies (KBr): $v_{\max } 1615$ (aromatic $\mathrm{C}=\mathrm{C}$ ), 1674 (C=O, $\delta$-lactone), 3055 (aromatic $\mathrm{C}-\mathrm{H}$ ), 3300 (broad, -OH) cm ${ }^{-1}$; ${ }^{1} \mathrm{H}-\mathrm{NMR}\left(400 \mathrm{MHz} ; \mathrm{CDCl}_{3}\right.$ ) $\delta_{\mathrm{ppm}}: 7.2-8.0$ $(13 \mathrm{H}, \mathrm{m}, \mathrm{Ar}-\mathrm{H}), 11.49\left(1 \mathrm{H}, \mathrm{s},-\mathrm{OH}\right.$ proton, $\mathrm{D}_{2} \mathrm{O}$ exchangeable); ${ }^{13} \mathrm{C}-\mathrm{NMR}\left(100 \mathrm{MHz} ; \mathrm{CDCl}_{3}\right) \delta_{\mathrm{ppm}}: 105.0(\mathrm{C}), 114.3(\mathrm{CH})$, $117.8(\mathrm{CH}), \quad 118.0(\mathrm{CH}), \quad 118.3(\mathrm{C}), \quad 123.4(\mathrm{CH}), \quad 125.2(\mathrm{CH})$, $125.3(\mathrm{CH}), \quad 125.3(\mathrm{CH}), \quad 126.2(\mathrm{CH}), 126.7(\mathrm{CH}), 126.8(\mathrm{CH})$, $128.5(\mathrm{CH}), \quad 128.9(\mathrm{CH}), \quad 130.7(\mathrm{CH}), \quad 130.8(\mathrm{C}), \quad 133.7(\mathrm{C})$, 135.0(C), 138.4(C), 150.4(C), 150.8(C), 162.3(C), 165.4(C=O of coumarin); Elemental analysis: $\mathrm{C}_{23} \mathrm{H}_{14} \mathrm{O}_{3}$ requires C, 81.64; H, 4.17\%. Found: C, 81.70; H, 4.21\%. MS m/z: $338.0(\mathrm{M}+$ ).

7-hydroxy-4-methoxy-9-(naphthalen-1-yl)-6Hbenzo[c]chromen-6-one (2b): Yield $=61 \%$ white crystalline solid. m.p. $=185-187{ }^{\circ} \mathrm{C}$. Selected IR frequencies (KBr): 1620 ( $\mathrm{C}=\mathrm{C}$, aromatic), $1678(\mathrm{C}=\mathrm{O}, \delta$-lactone), 3058 (aromatic C-H), 3431 (broad, -OH) cm ${ }^{-1}$; ${ }^{1} \mathrm{H}-\mathrm{NMR}(400 \mathrm{MHz}$; DMSO- $\left.d_{6}\right) \delta_{\mathrm{ppm}}: 3.95\left(3 \mathrm{H}, \mathrm{s},-\mathrm{OCH}_{3}\right), 7.19-8.07(12 \mathrm{H}, \mathrm{m}, \mathrm{Ar}-\mathrm{H})$, $11.35\left(1 \mathrm{H}, \mathrm{s},-\mathrm{OH}\right.$ proton, $\mathrm{D}_{2} \mathrm{O}$ exchangeable); ${ }^{13} \mathrm{C}-\mathrm{NMR}$ $\left(100 \mathrm{MHz} ; \mathrm{DMSO}-d_{6}\right) \delta_{\mathrm{ppm}}: 56.09\left(-\mathrm{OCH}_{3}\right), 105.06(\mathrm{CH})$, 114.98(CH), 115.39(CH), 117.82(CH), 118.59(C), 124.91(CH), 125.08(CH), $125.50(\mathrm{CH}), 125.82(\mathrm{C}), 126.20(\mathrm{CH}), 126.85(\mathrm{CH})$, 126.96(CH), 128.45(CH), 128.71(CH), 130.33(C), 133.33(C), 135.32(C), 137.96(C), 139.82(C), 144.80(C), 149.43(C), 161.10(C), 163.85( $\mathrm{C}=\mathrm{O}$ of coumarin); Elemental analysis: $\mathrm{C}_{14} \mathrm{H}_{16} \mathrm{O}_{4}$ requires C, 78.25; H, 4.38\%. Found: C, 78.28; H, 4.41\%. MS m/z: $368.0(\mathrm{M}+)$.

4-hydroxy-2-(naphthalen-1-yl)-5H-dibenzo[c,f] chromen-5-one (2c): Yield $=72 \%$ white crystalline solid. m.p. $=194-196{ }^{\circ} \mathrm{C}$. Selected IR frequencies (KBr): 1618 ( $\mathrm{C}=\mathrm{C}$, aromatic), $1678(\mathrm{C}=\mathrm{O}, \delta$-lactone), 3048 (aromatic $\mathrm{C}-\mathrm{H}$ ), 3411 (broad, -OH) cm ${ }^{-1}$; ${ }^{1} \mathrm{H}-\mathrm{NMR}$ (400 MHz; DMSO$\left.d_{6}\right) \delta_{\mathrm{ppm}}: 7.2-8.9(15 \mathrm{H}, \mathrm{m}, \mathrm{Ar}-\mathrm{H}), 11.45(1 \mathrm{H}, \mathrm{s},-\mathrm{OH}$ proton, $\mathrm{D}_{2} \mathrm{O}$ exchangeable); ${ }^{13} \mathrm{C}$-NMR (100 MHz; DMSO- $\left.d_{6}\right) \delta_{\mathrm{ppm}}$ : 106.5(C), 112.6(C), 117.4(C), 117.5(C), 117.6(CH), 119.2(CH), 125.0(CH), $125.2(\mathrm{CH}), \quad 126.1(\mathrm{CH}), 126.2(\mathrm{CH}), 126.7(\mathrm{CH})$, 127.4(CH), $128.8(\mathrm{CH}), 129.0(\mathrm{CH}), 129.3(\mathrm{CH}), 129.9(\mathrm{CH})$, 130.7(C), 131.9(C), 132.8(CH), 133.9(C), 135.7(C), 138.4(C), 149.4(C), 150.1(C), 161.7 $\left(\mathrm{C}_{4}\right), 164.3(\mathrm{C}=\mathrm{O}$ of coumarin); Elemental analysis: $\mathrm{C}_{27} \mathrm{H}_{16} \mathrm{O}_{3}$ requires $\mathrm{C}, 83.49 ; \mathrm{H}, 4.15 \%$. Found: C, 83.51; H, 4.18\%. MS m/z: 388.41 (M+).

7-hydroxy-9-(naphthalen-2-yl)-6H-benzo[c] chromen-6-one (2d): Yield $=69 \%$ white crystalline solid. m.p. $=222-214^{\circ} \mathrm{C}$. Selected IR frequencies $(\mathrm{KBr})$ : $1614(\mathrm{C}=\mathrm{C}$, aromatic), 1681 ( $\mathrm{C}=\mathrm{O}, \delta$-lactone), 3109 (aromatic C-H), 3400 (broad, -OH) cm-1; ${ }^{1} \mathrm{H}-\mathrm{NMR}(400 \mathrm{MHz}$; $\left.\mathrm{CDCl}_{3}\right) \delta_{\mathrm{ppm}}: 7.28-8.21(13 \mathrm{H}, \mathrm{m}, \mathrm{Ar}-\mathrm{H}), 11.45(1 \mathrm{H}, \mathrm{s},-\mathrm{OH}$ proton, $\mathrm{D}_{2} \mathrm{O}$ exchangeable); ${ }^{13} \mathrm{C}-\mathrm{NMR}\left(100 \mathrm{MHz} ; \mathrm{CDCl}_{3}\right)$ $\delta_{\mathrm{ppm}}: 104.9(\mathrm{C}), 111.3(\mathrm{C}), 115.2(\mathrm{CH}), 117.8(\mathrm{CH}), 118.4(\mathrm{CH})$, $123.4(\mathrm{CH}), 125.0(\mathrm{CH}), 125.2(\mathrm{CH}), 126.7(\mathrm{CH}), 126.8(\mathrm{CH})$, $126.9(\mathrm{CH}), 127.7(\mathrm{CH}), 128.5(\mathrm{CH}), 128.9(\mathrm{CH}), 130.8(\mathrm{CH})$, 133.3(C), 133.4(C), 135.5(C), 136.7(C), 150.2(C), 150.8(C), 162.6(C), 165.4( $\mathrm{C}=\mathrm{O}$ of coumarin); Elemental analysis: $\mathrm{C}_{23} \mathrm{H}_{14} \mathrm{O}_{3}$ requires $\mathrm{C}, 84.64 ; \mathrm{H}, 4.17 \%$. Found: $\mathrm{C}, 84.69 ; \mathrm{H}$, 4.21\%. MS m/z: $338.0(\mathrm{M}+)$.

7-hydroxy-4-methoxy-9-(naphthalen-2-yl)-6Hbenzo[c]chromen-6-one (2e): Yield $=59 \%$ white crystalline solid. m.p. $=208-210{ }^{\circ} \mathrm{C}$. Selected IR frequencies (KBr): $1617(\mathrm{C}=\mathrm{C}$, aromatic), $1682(\mathrm{C}=\mathrm{O}, \delta$-lactone), 3048 (aromatic C-H), 3400 (broad, - $\mathrm{OH}$ ) cm ${ }^{-1}$; ${ }^{1} \mathrm{H}-\mathrm{NMR}(400 \mathrm{MHz}$; DMSO- $\left.d_{6}\right) \delta_{\mathrm{ppm}}: 3.91\left(3 \mathrm{H}, \mathrm{s},-\mathrm{OCH}_{3}\right) 7.23-8.49(12 \mathrm{H}, \mathrm{m}, \mathrm{Ar}-\mathrm{H})$, $11.26\left(1 \mathrm{H}, \mathrm{s},-\mathrm{OH}\right.$ proton, $\mathrm{D}_{2} \mathrm{O}$ exchangeable); ${ }^{13} \mathrm{C}-\mathrm{NMR}$ $\left(100 \mathrm{MHz}\right.$; DMSO- $\left.d_{6}\right) \delta_{\mathrm{ppm}}$ : 56.7(- $\left.\mathrm{OCH}_{3}\right), 105.3(\mathrm{C}), 112.3(\mathrm{CH})$, 113.6(CH), $\quad 114.6(\mathrm{CH}), \quad 116.0(\mathrm{CH}), \quad 119.2(\mathrm{C}), \quad 125.4(\mathrm{CH})$, 125.5(CH), 127.1(CH), 127.4(CH), $128.0(\mathrm{CH}), 129.0(\mathrm{CH})$, 133.5(C), 133.5(C), 135.8(C), 136.2(C), 140.3(C), 147.8(C), 149.3(C), 162.1(C), 164.5(C=O of coumarin); Elemental analysis: $\mathrm{C}_{24} \mathrm{H}_{16} \mathrm{O}_{4}$ requires $\mathrm{C}, 78.25 ; \mathrm{H}, 4.38 \%$. Found: C, 78.28; H, 4.41\%. MS m/z: $368.0(\mathrm{M}+)$.

4-hydroxy-2-(naphthalen-2-yl)-5H-dibenzo[c,f] chromen-5-one (2f): Yield $=78 \%$ white crystalline solid. m.p. $=222-224{ }^{\circ} \mathrm{C}$. Selected IR frequencies (KBr): $1607(\mathrm{C}=\mathrm{C}$, aromatic), 1675 ( $\mathrm{C}=\mathrm{O}, \delta$-lactone), 3049 (aromatic C-H), 3565 (broad, -OH) $\mathrm{cm}^{-1}$; ${ }^{1} \mathrm{H}-\mathrm{NMR}(400 \mathrm{MHz}$; DMSO- $\left.d_{6}\right) \delta_{\mathrm{ppm}}: 7.37-8.97(15 \mathrm{H}, \mathrm{m}, \mathrm{Ar}-\mathrm{H}), 11.39(1 \mathrm{H}, \mathrm{s},-\mathrm{OH}$ proton, $\mathrm{D}_{2} \mathrm{O}$ exchangeable); ${ }^{13} \mathrm{C}$-NMR (100 MHz; DMSO$\left.d_{6}\right) \delta_{\mathrm{ppm}}: 106.4(\mathrm{C}), 112.8(\mathrm{C}), 114.6(\mathrm{CH}), 116.5(\mathrm{CH}), 117.6(\mathrm{CH})$, 125.4(CH), $125.5(\mathrm{CH}), \quad 126.3(\mathrm{CH}), \quad 127.1(\mathrm{CH}), 127.4(\mathrm{CH})$, 127.5(CH), $\quad 128.0(\mathrm{CH}), \quad 129.1(\mathrm{CH}), \quad 129.3(\mathrm{CH}), \quad 129.4(\mathrm{C})$, 129.9(CH), 132.0(C), 132.8(CH), 133.4(C), 133.6(C), 136.2(C), 136.5(C), 148.3(C), 149.3(C), 150.1(C), 162.1(C), 164.5(C=O of coumarin); Elemental analysis: $\mathrm{C}_{27} \mathrm{H}_{16} \mathrm{O}_{3}$ requires $\mathrm{C}$, 83.49; H, 4.15\%. Found: C, 83.52; H, 4.19\%. MS m/z: $388.0(\mathrm{M}+$ ).

It is important to note that in the case of compound 2e, the number of non-equivalent carbon signals in ${ }^{13} \mathrm{C}$ NMR spectra is less than expected (two signals). This may be due to identical chemical shifts of certain carbon atoms which may appear at the same position.

\section{Anti-bacterial activity}

All the synthesized compounds (2a-2f) were screened for their antibacterial activity using the Broth microdilution method. The synthesized compounds were investigated to 
determine their antibacterial activity in terms of minimum inhibitory concentration (MIC) using the serial dilution method [21]. Concentrations between $0.20,0.40,0.80$, $1.60,3.12,6.25,12.5,25,50$ and $100 \mu \mathrm{g} / \mathrm{ml}$ of each active compound were tested and compared with a standard drug. The MIC was then determined as the lowest concentration inhibiting the growth of the organism after 24-48 h.

\section{Cytotoxicity}

The tested human carcinoma cell lines were obtained from the NCCS Pune. The cells were maintained at $37{ }^{\circ} \mathrm{C}$ in appropriate media and humidified atmosphere with $5 \% \mathrm{CO}_{2}$ For cytotoxicity assay, cancer cell lines were suspended in the medium at cell density $5 \times 10^{4}$ cells/well in 96-well tissue culture plates, then incubated overnight. The tested compounds were added to 96-well plates (3 replicates) to achieve different concentrations $(5,10,25$, $50,100,200,300 \mu \mathrm{M})$ for each compound. Three vehicle controls with media or $0.5 \%$ DMSO were run for each 96-well plate as a control. After incubating the treated cells for $24 \mathrm{~h}$, the numbers of viable cells were determined by the MTT assay [22]. Rapid colorimetric assay was used for determination of cellular growth and cell-survival. This had application to proliferation and cytotoxicity assays.

The relationship between surviving cells and drug concentration is plotted to get the survival curve for each cell line after treatment with the specified compound. The $50 \%$ inhibitory concentration $\left(\mathrm{IC}_{50}\right)$, the concentration required to cause toxic effects in $50 \%$ of intact cells, was estimated by nonlinear regression analysis using Graph pad prism (version 7.0) software and expressed in mean \pm SD.

Acknowledgements: The authors express their sincere thanks to the Department of Advanced Organic Chemistry, P. D. Patel Institute of Applied Sciences, Charotar University of Science \& Technology (CHARUSAT) for providing research facilities.

\section{References}

[1] Borges, F.; Roleira, F.; Milhazes, N.; Santana, L.; Uriarte, E. Simple Coumarins and Analogues in Medicinal Chemistry: Occurrence, Synthesis and Biological Activity. Curr. Med. Chem. 2005, 12, 887-916.

[2] Laurin, P.; Ferroud, D.; Klich, M.; Dupuis-Hamelin, C.; Mauvais, P.; Lassaigne, P.; Bonnefoy, A.; Musicki, B. Synthesis and in Vitro Evaluation of Novel Highly Potent Coumarin Inhibitors of Gyrase B. Bioorg. Med. Chem. Lett. 1999, 9, 2079-2084.
[3] Zhang, L.; Jiang, G.; Yao, F.; He, Y.; Liang, G.; Zhang, Y.; Hu, B.; Wu, Y.; Li, Y.; Liu, H. Growth Inhibition and Apoptosis Induced by Osthole, A Natural Coumarin, in Hepatocellular Carcinoma. PLoS One 2012, 7, e37865.

[4] Lee, S.-Y.; Lim, T.-G.; Chen, H.; Jung, S. K.; Lee, H.-J.; Lee, M.-H.; Kim, D. J.; Shin, A.; Lee, K. W.; Bode, A. M.; et al. Esculetin Suppresses Proliferation of Human Colon Cancer Cells by Directly Targeting $\beta$-Catenin. Cancer Prev. Res. (Phila). 2013, 6, 1356-1364.

[5] Ostrov, D. A.; Hernandez Prada, J. A.; Corsino, P. E.; Finton, K. A.; Le, N.; Rowe, T. C. Discovery of Novel DNA Gyrase Inhibitors by High-Throughput Virtual Screening. Antimicrob. Agents Chemother. 2007, 51, 3688-3698.

[6] Riveiro, M. E.; Moglioni, A.; Vazquez, R.; Gomez, N.; Facorro, G.; Piehl, L.; de Celis, E. R.; Shayo, C.; Davio, C. Structural Insights into Hydroxycoumarin-Induced Apoptosis in U-937 Cells. Bioorg. Med. Chem. 2008, 16, 2665-2675.

[7] Zhang, Y.; Zou, B.; Chen, Z.; Pan, Y.; Wang, H.; Liang, H.; Yi, X. Synthesis and Antioxidant Activities of Novel 4-Schiff Base-7Benzyloxy-Coumarin Derivatives. Bioorg. Med. Chem. Lett. 2011, 21, 6811-6815.

[8] Curini, M.; Epifano, F.; Maltese, F.; Marcotullio, M. C.; Gonzales, S. P.; Rodriguez, J. C. Synthesis of Collinin, an Antiviral Coumarin. Aust. J. Chem. 2003, 56, 59-60.

[9] Manvar, A.; Bavishi, A.; Radadiya, A.; Patel, J.; Vora, V.; Dodia, N.; Rawal, K.; Shah, A. Diversity Oriented Design of Various Hydrazides and Their in Vitro Evaluation against Mycobacterium Tuberculosis H37Rv Strains. Bioorg. Med. Chem. Lett. 2011, 21, 4728-4731.

[10] Matos, M. J.; Terán, C.; Pérez-Castillo, Y.; Uriarte, E.; Santana, L.; Viña, D. Synthesis and Study of a Series of 3-Arylcoumarins as Potent and Selective Monoamine Oxidase B Inhibitors. J. Med. Chem. 2011, 54, 7127-7137.

[11] Sidwell, W. T. L.; Fritz, H.; Tamm, C. Autumnariol Und Autumnariniol, Zwei Neue Dibenzo-?-Pyrone AusEucomis Autumnalis Graeb. Nachweis Einer Fernkopplung ber Sechs Bindungen in Den Magnetischen Protonenresonanz - Spektren. Helv. Chim. Acta 1971, 54, 207-215.

[12] Raistrick, H.; Stickings, C. E.; Thomas, R. Studies in the Biochemistry of Microorganisms. 90. Alternariol and Alternariol Monomethyl Ether, Metabolic Products of Alternaria Tenuis. Biochem. J. 1953, 55, 421-433.

[13] Pero, R. W.; Harvan, D.; Blois, M. C. Isolation of the Toxin, Altenuisol, from the Fungus, Alternaria Tenuis Auct. Tetrahedron Lett. 1973, 14, 945-948.

[14] Raistrick, H.; Stilkings, C. E.; Thomas, R. ALTERNARIOL AND ALTERNARIOL MONOMETHYL ETHER, METABOLIC PRODUCTS OF ALTERNARIA TENUIS. Biochem. J. 1953, 55, 421-433.

[15] Giorgio, C.; Alessandra, G.; Paolo, B.; Elisa, Z.; Riccardo, P.; Angelo, P.; Lorenzo, A. P., Flavio, M.; Giuseppe, Z.; Stefano, M. Urolithin as a Converging Scaffold Linking Ellagic acid and Coumarin Analogues: Design of Potent Protein Kinase CK2 Inhibitors. ChemMedChem. 2011, 12, 2273-2286.

[16] Álvarez, C.; Álvarez, R.; Corchete, P.; Pérez-Melero, C.; Peláez, R.; Medarde, M. Synthesis and Biological Activity of Naphthalene Analogues of Phenstatins: Naphthylphenstatins. Bioorg. Med. Chem. Lett. 2007, 17, 3417-3420.

[17] Budhiraja, A.; Kadian, K.; Kaur, M.; Aggarwal, V.; Garg, A.; Sapra, S.; Nepali, K.; Suri, O. P.; Dhar, K. L. Synthesis and Biological Evaluation of Naphthalene, Furan and Pyrrole Based 
Chalcones as Cytotoxic and Antimicrobial Agents. Med. Chem. Res. 2012, 21 (9), 2133-2140.

[18] Mario, C. F.; Erin, R. J.; Melinda, R.V.; James, S. W.; Ross, L.; Barclay, C.; Ingold, K. U. Naphthalene Diols: A New Class of Antioxidants Intramolecular Hydrogen Bonding in Catechols, Naphthalene Diols, and Their Aryloxyl Radicals. Journal of Organic Chemistry. 2002, 67, 5190-5196.

[19] Pandya, A.; Prajapati, D.; Pandya, S. Synthesis of novel Naphthalene COX inhibitors for anti-inflammatory activity. Journal of Applied Pharmaceutical Science. 2012, 2, 226-232.

[20] Kaushal P. Patel. A One Pot Synthesis of Fused Chromenones. ARKIVOC 2013, 2013, 14-23.

[21] Balows, A. Color Atlas and Textbook of Diagnostic Microbiology. Diagn. Microbiol. Infect. Dis. 1985, 3 (3), 274-275.

[22] Mosmann, T. Rapid Colorimetric Assay for Cellular Growth and Survival: Application to Proliferation and Cytotoxicity Assays. J. Immunol. Methods 1983, 65, 55-63. 